\title{
Measurements to Determine the Regimes of Premixed Flames in Extreme Turbulence
}

\author{
Timothy M. Wabel ${ }^{\mathrm{a}, *}$, Aaron W. Skiba ${ }^{\mathrm{a}}$, Jacob E. Temme ${ }^{\mathrm{b}}$, James F. Driscoll ${ }^{\mathrm{c}}$ \\ ${ }^{a}$ Graduate Student Research Assistant, University of Michigan, Ann Arbor, MI 48109 USA \\ ${ }^{b}$ U.S. Army Research Laboratory, Aberdeen Proving Ground, MD 21005, USA \\ ${ }^{c}$ Arthur B. Modine Professor, University of Michigan, Ann Arbor, MI 48109 USA
}

\begin{abstract}
A new regime of extreme turbulence - defined as the ratio of turbulence intensity to laminar flame speed $u^{\prime} / S_{L}$ from 25 to 243 - was characterized for six premixed flames using a new piloted Bunsen burner (called Hi-Pilot). The flames studied had $u^{\prime} / S_{L}$ values several times larger than those of previous related studies and integral scales and turbulent Reynolds numbers as large as $41 \mathrm{~mm}$ and 99,000, respectively. Layer thicknesses were determined from planar laser-induced fluorescence (PLIF) images of $\mathrm{OH}$ and formaldehyde. Preheat layer thickness was found to increase to sixteen times the laminar value. Residence time of eddies in the flame appears to be important, since the flame tip had preheat regions that were thicker than at the flame base. Reaction layers were not broadened, remaining below twice the laminar value. Four of the cases were predicted to lie in the Broadened Preheat - Thin Reaction layer (BP-TR) regime and the measurements confirmed that they had a BP-TR structure. However, two cases went far beyond the predicted boundary for the Broken Reactions (BR) regime but measurements showed that they were not broken but retained their BP-TR structure. Thus the regime of BP-TR is measured to persist over a wider range than previously predicted. One explanation is that the turbulent eddies may become weakened by the thick, viscous preheat layer before they arrive at the reaction front. Distributed reactions were not observed in the six cases that were selected.
\end{abstract}

Keywords: Turbulent Combustion, Regime Diagram, PLIF

\section{Introduction}

This work addresses the need to experimentally measure the boundaries between the different regimes of premixed turbulent combustion. Such boundaries are obtained from scaling analyses and are typically combined to form regime

\footnotetext{
* Corresponding author

Email address: twabel@umich.edu (Timothy M. Wabel)
} 
diagrams. An ideal regime diagram should be capable of predicting the flame structure based on a condensed set of known parameters. One motivation for a regime diagram is that a realistic design model should employ a flamelet-like sub-model [1] when the operating conditions lie within the flamelet regime, while a "well-stirred" sub-model (that might contain auto-ignition chemistry) would be a better choice to simulate Homogeneous Charge Compression Ignition (HCCI) engine conditions that lie in the distributed reaction regime. Several numerical simulations have considered different regimes (Sankaran et al. [2], Aspden et al. [3], Savard et al. [4], Sundaram et al. [5], Huh et al. [6] and Lee and Huh [7]) but experimental verification is lacking.

Previously, Gülder et al. [8, 9] performed planar Rayleigh scattering and hydroxyl $(\mathrm{OH})$ planar laser-induced fluorescence (PLIF) imaging of premixed Bunsen flames for the largest turbulence levels at that time. They achieved $u^{\prime} / S_{L}$ values (where $u$ ' is the root-mean-square of turbulent fluctuations and $S_{L}$ is the laminar flame speed) as large as 24 . The present work considers the new range of extreme turbulence in Bunsen geometries, defined as $u^{\prime} / S_{L} \geq$ 25. Relevant experimental studies in other geometries have been performed by Bradley, Mansour et al. [10, 11], Dinkelacker et al.[12, 13], and Kariuki et al. [14].

The Borghi regime diagram [15] is shown in Figure 1, along with the present measurement points. When placing the test cases on the regime diagram, flame thickness is defined following Peters $[16,17]$ as $\delta_{F}=D^{*} / S_{L}$, where $D^{*}$ is evaluated at an elevated reaction zone temperature $T^{*}$. Here, $T^{*}=1,500 \mathrm{~K}$ and $D^{*}=7.2 \times 10^{-5} \mathrm{~m}^{2} / \mathrm{s}$. There remain two interesting and relatively unexplored areas on the Borghi diagram in Figure 1. The upper left region signifies large Karlovitz numbers, and has been the subject of numerous DNS studies $[3,4]$. The upper right region corresponds to large turbulent Reynolds numbers $\left(R e_{T}=u^{\prime} L_{x} / \nu\right.$, where $L_{x}$ is the longitudinal integral scale and $\nu$ is the kinematic viscosity) and is the subject of the present study. The boundary marked "K-W" is the Klimov-Williams limit where Kolmogorov eddies fit inside the preheat layer $[16,18]$. As this boundary is crossed the preheat layer is predicted to broaden, but the reaction layer is not expected to deviate from the laminar thickness. Therefore, it is called the Broadened Preheat - Thin Reaction layer (BP-TR) regime. Peters [16] predicts that broken reactions may occur when Kolmogorov eddies fit inside the reaction layer. This boundary is marked "BR" in Figure 1. Distributed reactions have been predicted to occur in either the upper right or upper left regions, but minimal useful experimental information is available. Images of reaction layers by Dunn et al. [19] and Zhou et al. [20] provide the first evidence using PLIF diagnostics that broadening and some distributed reactions occur. Previously there have been several efforts to provide experimental regime information [19, 20, 21, 22, 23, 24, 25]. Rayleigh scattering was used to measure flamelet thermal thicknesses by Sweeney et al. [21], Mansour et al. [22] and others [23, 24, 25]. DNS studies [3, 4] were performed at large $K a_{T}(>1,500)$ but small $\operatorname{Re}_{T}(<55)$ by selecting small $(1 \mathrm{~mm})$ integral scales, with differing results. Savard et al. [4] observed broken reaction zones (in agreement with the predicted BR boundary) due to the high strain rates; 


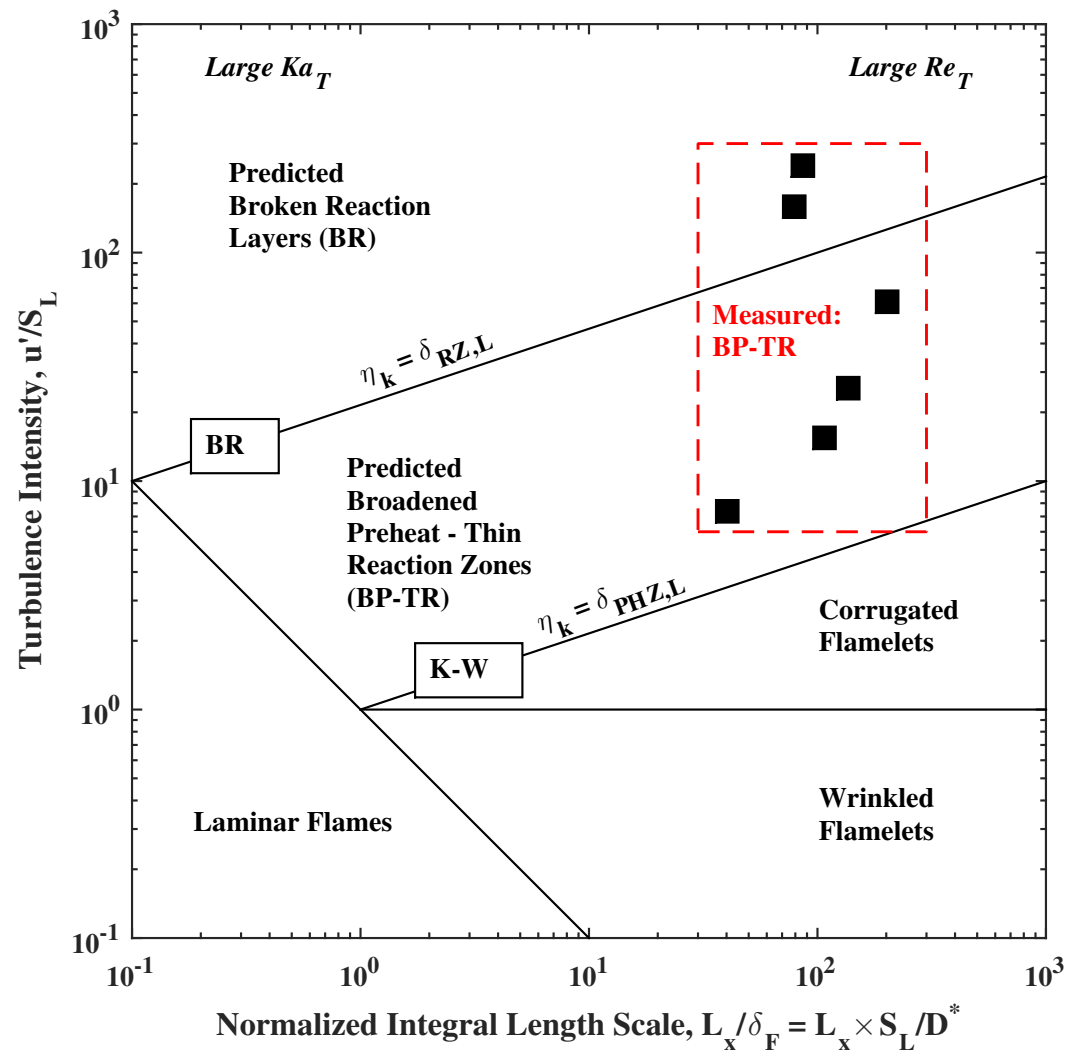

Figure 1: Borghi regime diagram as predicted by Peters [15, 16, 17]. Theoretical regime boundaries are labeled K-W (Klimov-Williams) and BR (Broken Reactions). Square symbols indicate the present measurements.

however Aspden et al. [3] studied flames subjected to similar turbulence and reported evidence of distributed burning. Therefore, whether combustion occurs as broken or distributed reactions as the predicted BR boundary is crossed is unclear.

\section{Calibration of the PLIF Diagnostics}

The reaction and preheat zones were imaged using simultaneous $\mathrm{OH}$ and formaldehyde $\left(\mathrm{CH}_{2} \mathrm{O}\right)$ PLIF diagnostics that are described in Ref. [26]. An $\mathrm{Nd}$ :YAG and an Nd:YAG-pumped dye laser was operated at $355 \mathrm{~nm}$ and 284.07 $\mathrm{nm}$ to excite $\mathrm{CH}_{2} \mathrm{O}$ and $\mathrm{OH}$, respectively, and fluorescence was collected by intensified, gated, UV sensitive cameras. $\mathrm{Li}$ et al. [27] has shown that $\mathrm{CH}_{2} \mathrm{O}$ 
can be used as a marker of the preheat layer. The reaction zone is determined by the Overlap method $[27,28,29,30,31,32]$, wherein the Overlap is defined as the product of the $\mathrm{OH}$ and $\mathrm{CH}_{2} \mathrm{O}$ PLIF images. Arrhenius kinetics require that reactions between $\mathrm{OH}$ and $\mathrm{CH}_{2} \mathrm{O}$ must occur in the Overlap region, but this is only one of several possible reaction layers. Reaction layer thickness is defined to be the full width at half maximum of the Overlap region. Local extinction (broken reactions) is defined to occur when the Overlap signal drops below this threshold. The flame was first imaged using a large field of view (FOV) of approximately $150 \times 150 \mathrm{~mm}^{2}$ to qualitatively visualize the flame structure. In order to obtain accurate Overlap measurements, a smaller FOV providing high resolution was required. The FOV for these measurements was $30 \times 30 \mathrm{~mm}^{2}$, and the spatial resolution (derived from the binned pixel size and laser sheet thickness) was $59 \times 59 \times 200 \mu^{3}$.

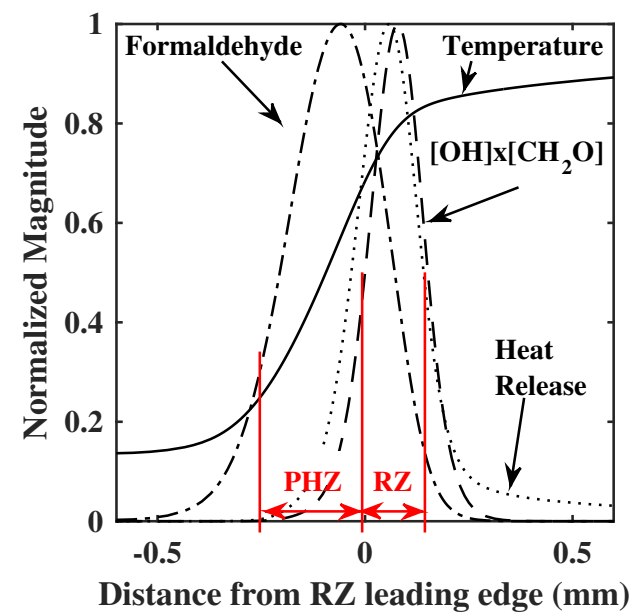

Figure 2: CHEMKIN-based laminar flame computations for methane-air, equivalence ratio of 1.05 , showing that $\mathrm{CH}_{2} \mathrm{O}$ marks the preheat zone while the overlap of $\mathrm{CH}_{2} \mathrm{O}-\mathrm{OH}$ signal marks the reaction zone. Vertical lines mark the preheat and reaction zones.

To assess the experimental approach, calibration studies were conducted with both a laminar conical Bunsen flame and CHEMKIN computations. The CHEMKIN simulation profiles (for methane-air and equivalence ratio of 1.05 , Figure 2) show that the heat release rate profile nearly coincides with the Overlap profile; however, it should be noted that the PLIF signals are uncorrected for electronic quenching or Boltzmann population fraction. The simulations also show that $\mathrm{CH}_{2} \mathrm{O}$ exists throughout the preheat zone because it diffuses upstream from the reaction zone at the same rate as the gas temperature. The leading edge of the preheat zone is defined to be where $\mathrm{CH}_{2} \mathrm{O}$ concentration is $35 \%$ of its maximum, and CHEMKIN simulations indicate that the gas temperature here is $550 \mathrm{~K}$. The trailing edge of the preheat zone is where the reaction zone begins. Laminar flame thickness is the sum of preheat and reaction zone 
thicknesses $\left(\delta_{F, L}=\delta_{P H Z, L}+\delta_{R Z, L}\right)$ and is $0.39 \mathrm{~mm}$ for $\phi=1.05$. This agrees with the thermal thickness computed using the CHEMKIN temperature profile, $\left(T_{\max }-T_{\min }\right) /\left.(d T / d x)\right|_{\max }=0.43 \mathrm{~mm}$.

The laminar calibration flame data showed that in the overlap region the product of the $\mathrm{OH}$ and $\mathrm{CH}_{2} \mathrm{O}$ PLIF signals was a Gaussian profile that had a peak value that was constant and continuous along the flame. The typical signal-to-noise ratio was 130 for $\mathrm{CH}_{2} \mathrm{O}$ and 250 for $\mathrm{OH}$ after median and levelset filtering, and 33 for the processed Overlap images. Here, "noise" is defined to be the R.M.S. signal fluctuations in the laminar flame where the $\mathrm{OH}$ and $\mathrm{CH}_{2} \mathrm{O}$ concentrations are uniform. Table 1 lists the measured layer thicknesses from the Bunsen flame compared to the CHEMKIN simulations.

Table 1: Measured and simulated laminar layer thicknesses in millimeters

\begin{tabular}{cccc}
\hline & & $\phi=1.05$ & $\phi=0.65$ \\
\hline \multirow{2}{*}{$\delta_{R Z}$} & Measured & 0.36 & 0.52 \\
& CHEMKIN & 0.15 & 0.22 \\
\multirow{2}{*}{$\delta_{P H Z}$} & Measured & 0.39 & 0.45 \\
& CHEMKIN & 0.24 & 0.49 \\
\hline
\end{tabular}

\section{Michigan Hi-Pilot Burner and Operating Conditions}

The Michigan Hi-Pilot burner [26] shown in Figure 3 is a piloted Bunsen burner that is designed to achieve very large values of $u^{\prime} / S_{L}, L_{x}$, and $R e_{T}$, along with a uniform profile of $u$ ' and $L_{x}$. Hotwire anemometer measurements showed that u'varied by less than $15 \%$ across the central $80 \%$ of the burner exit with no flame present. Reactants issue at mean velocities up to $U_{0}=78$ $\mathrm{m} / \mathrm{s}$ from the central burner that is $21.6 \mathrm{~mm}$ in diameter. The conical premixed flame is located near the downstream edge of the jet core that has a height of about 6 burner diameters $(126 \mathrm{~mm})$ [33]. Within this jet core, $u^{\prime}$ and $L_{x}$ are estimated to be relatively constant. The turbulence generator [34] consists of a slotted plate upstream of a converging nozzle. Longitudinal integral scales $\left(L_{x}\right)$ were measured by a laser Doppler velocimeter. To validate the integral scale measurement, $L_{x}$ was evaluated in a pipe flow and found to agree with published values. Further details on the integral scale measurements are found in [35]. Conditions for all of the methane-air flames described in this paper are given in Table 2. Cases $2 \mathrm{a}-5 \mathrm{a}$ employed slightly rich mixtures with equivalence ratio $\phi=1.05$. A lean flame with $\phi=0.65$ was also studied for Cases 5a-6a.

\section{Results}

4.1. Preheat Structure for Extreme Turbulence $\left(u^{\prime} / S_{L}\right.$ up to 243)

It is illustrative to begin by presenting the large FOV PLIF results, which provide a qualitative sense of the flame structure. Figure 4 shows instantaneous preheat zones (blue) in the Hi-Pilot burner for Cases 2-5a $(\phi=1.05)$, together 
Table 2: Run conditions and parameters for the Hi-Pilot Experiment

\begin{tabular}{ccccccccc}
\hline Case & $\phi$ & $U_{0}(\mathrm{~m} / \mathrm{s})$ & $u^{\prime}(\mathrm{m} / \mathrm{s})$ & $L_{x}(\mathrm{~mm})$ & $R e_{T}$ & $D a_{T}$ & $u^{\prime} / S_{L}$ & $L_{x} / \delta_{P H Z, L}$ \\
\hline $2 \mathrm{a}$ & 1.05 & 14 & 2.9 & 7.5 & 1,440 & 25 & 7.5 & 31 \\
$3 \mathrm{a}$ & 1.05 & 32 & 6.0 & 20 & 7,900 & 33 & 15 & 84 \\
$4 \mathrm{a}$ & 1.05 & 44 & 10 & 25 & 16,500 & 25 & 26 & 105 \\
$5 \mathrm{a}$ & 1.05 & 64 & 24 & 37 & 58,200 & 15 & 62 & 154 \\
$5 \mathrm{a}$ & 0.65 & 64 & 24 & 37 & 58,200 & 2.3 & 157 & 75 \\
$6 \mathrm{a}$ & 0.65 & 78 & 37 & 41 & 99,000 & 1.7 & 243 & 83 \\
\hline
\end{tabular}

with $\mathrm{OH}$ images (red) and the Overlap (yellow) signal. Preheat layers for Case $2 \mathrm{a}$ are continuous and in regions near the burner exit have a thickness that is close to the measured laminar value $\left(\delta_{P H Z, L}\right)$ of $0.39 \mathrm{~mm}$. For Cases $3-5 \mathrm{a}$ it is surprising to see the extremely thick preheat zones in Figures $4 \mathrm{c}$ and $4 \mathrm{~d}$. To the authors' knowledge, preheat zones this thick have not been measured before. Case $4 \mathrm{a}$, for example, shows a preheat thickness $\delta_{P H Z, T}$ (indicated by the white arrow in frame c) of $20 \mathrm{~mm}$, which is more than ten times the laminar value. Figure 4 shows that $\delta_{P H Z, T}$ tends to increase with $u^{\prime} / S_{L}$ and with height (x) above the burner. Zhou et al. [20] found similar trends. This suggests that residence time (which scales as $x / U$ ) is an important parameter, since $\delta_{P H Z, T}$ increases with $(x / U)$ even though all locations experience about the same turbulence level and integral scales. This implies that some nondimensional residence time should be considered when constructing a regime diagram.

Average preheat thicknesses were evaluated using the smaller $\left(30 \times 30 \mathrm{~mm}^{2}\right)$ FOV, and measured by fitting a skeleton line that runs along each layer, halfway between the two edges. The distance between inner and outer boundaries is measured as twice the distance from each skeleton point to the nearest $\mathrm{CH}_{2} \mathrm{O}$ edge (defined as the point where formaldehyde signal drops below $35 \%$ of the local maximum). Because the trailing edge of the preheat zone is defined as the start of the reaction layer, binarized Overlap images (described in 4.2) were used to set all $\mathrm{CH}_{2} \mathrm{O}$ signal within the Overlap region to zero. As shown in Figure $5, \delta_{P H Z, T}$ values reach sixteen times the laminar value $\left(\delta_{P H Z, L}\right)$. All cases fall within the "broadened" preheat zone regime, defined as $\delta_{P H Z, T} / \delta_{P H Z, L}>2$. The KlimovWilliams criterion has often been cited as the boundary separating corrugated flamelets from Broadened Preheat - Thin Reaction layers (BP-TR) [16]. This criterion is based on the assumption that the preheat layer will broaden as the Kolmogorov scale of turbulence becomes small enough to penetrate the flame $\left(\eta_{k}<\delta_{P H Z, L}\right)$. In this regime, it is thought that the reaction layer will remain unaffected by the turbulence, and retain laminar flame properties. The present study does not consider conditions below the Klimov-Williams criterion (corrugated flamelets), and therefore cannot evaluate the boundary conclusively. However, it is observed that all four of the flames which fall in the predicted BP-TR regime agree with the predictions of Peters. Thus, the present data supports (but does not validate) the Klimov-Williams criterion. 


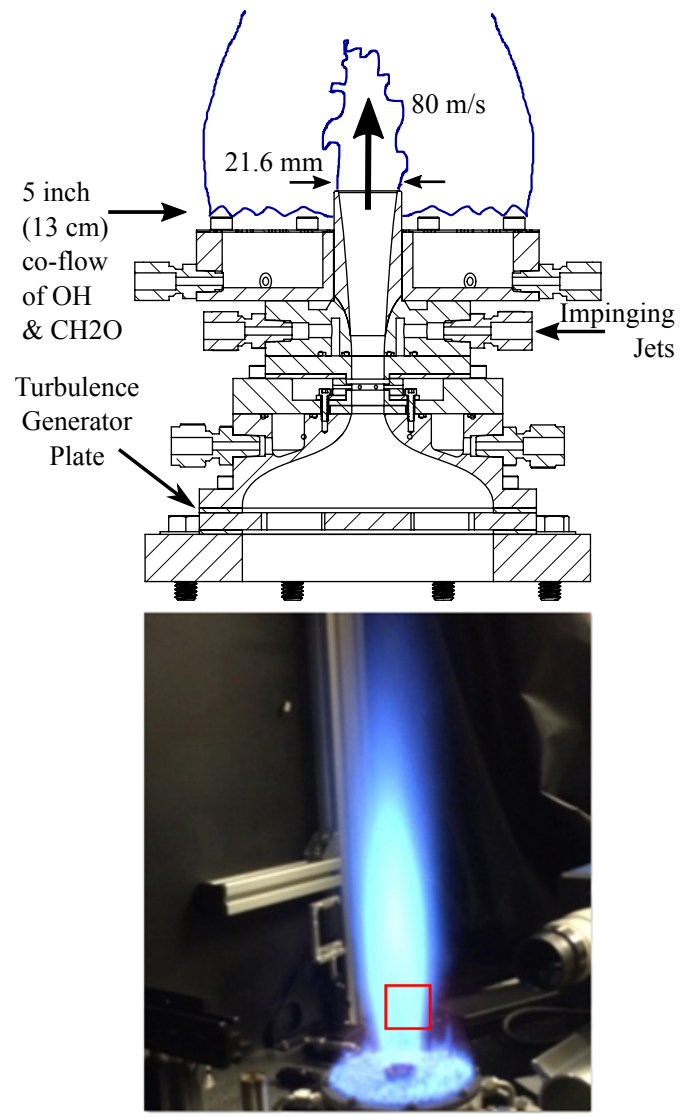

Figure 3: Hi-Pilot Burner operating with Case 2a. The interrogation region for high resolution imaging (described in Section 4) is indicated by the red box.

\subsection{Reaction zone structure}

Full-FOV Overlap reaction zones are shown as yellow regions in Figure 4 for Cases 2-5a $(\phi=1.05)$. For Case $2 \mathrm{a}$ there is significant large-scale wrinkling but no evidence of broken or distributed reactions. Case 5a also shows continuous and thin reaction layers despite a significant increase in $u^{\prime} / S_{L}$. Reaction zone thicknesses were obtained by imaging the smaller FOV $\left(30 \times 30 \mathrm{~mm}^{2}\right)$, permitting the high resolution required for an accurate Overlap measurement. Typical reaction layers for Cases 2a and 6a are given in Figure 6. For Case 6a there are few broken reaction layers and no significant degree of thickening is observed. It should be noted that local extinction of the Overlap signal does not necessarily imply that all reactions have ceased; rather the signal has simply dropped below the threshold used here (equaling $50 \%$ of the local maximum).

Time-averaged reaction layer thicknesses were computed similar to the preheat thicknesses by fitting a skeleton to each layer and measuring from the skele- 

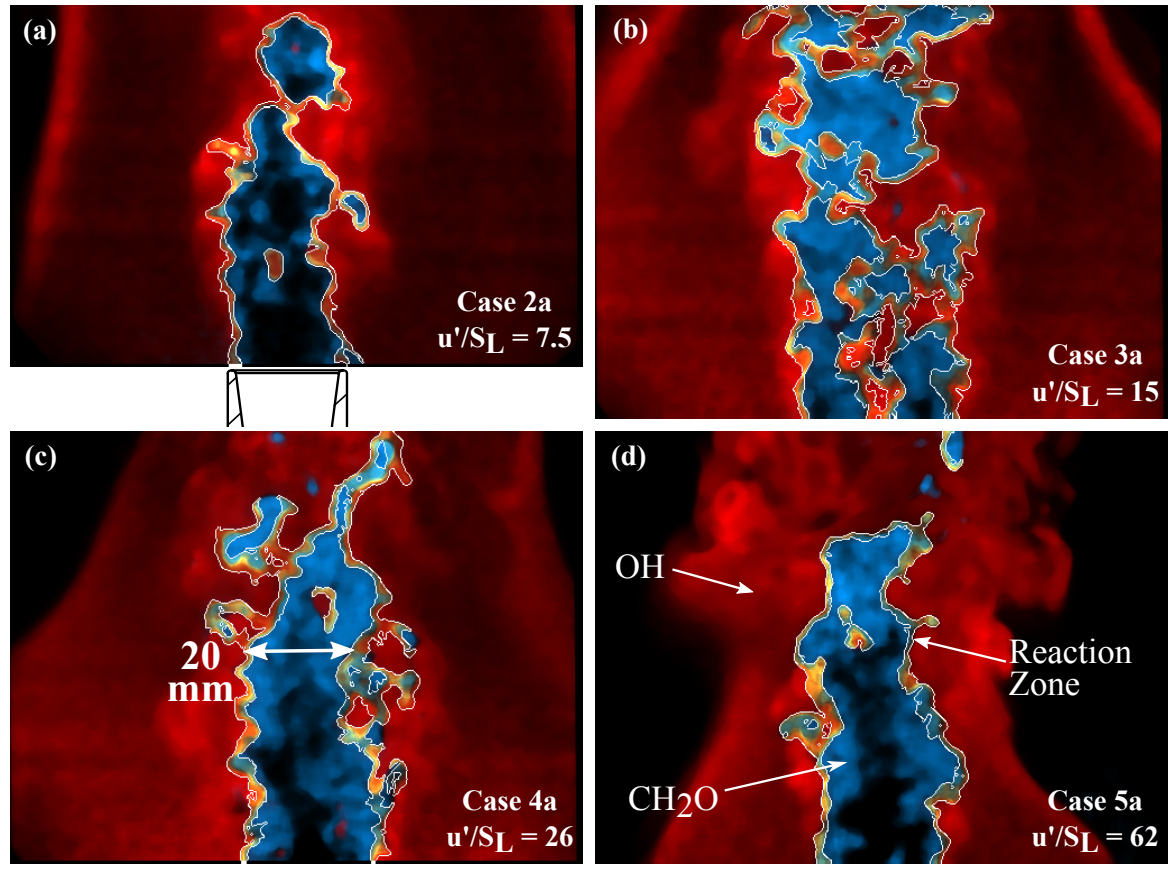

Figure 4: Simultaneous preheat zone $\left(\mathrm{CH}_{2} \mathrm{O}\right.$, blue), reaction zone (yellow), and $\mathrm{OH}$ (red) instantaneous images for $u^{\prime} / S_{L}$ from 7.5 to $62(\phi=1.05)$. White arrow in (c) indicates a preheat zone that is 50 times thicker than the laminar value $\left(\delta_{P H Z, L}\right)$ of $0.39 \mathrm{~mm}$. FOV in (a) is $69 \mathrm{~mm}$ (height) $\times 79 \mathrm{~mm}$, in (b)-(c) it is $90 \mathrm{~mm} \times 105 \mathrm{~mm}$, and in (d) it is $115 \mathrm{~mm} \times$ $130 \mathrm{~mm}$

ton to the nearest point where Overlap signal has decayed to $50 \%$ of the nearest local maximum (twice this distance gives the full-width at half-maximum). Reaction layers are called "thickened" if their average thickness exceeds twice the laminar value. Figure 5 shows that the normalized reaction zone thickness $\left(\delta_{R Z, T} / \delta_{R Z, L}\right)$ does not exceed two, indicating no significant broadening. Even at extreme turbulence of $u^{\prime} / S_{L}=243\left(R e_{T}=99,000\right)$, no substantial increase in reaction layer thickness was observed. The percentage of flamelet-like reaction zones was also calculated. We define a structure to be partially-distributed when the average normalized reaction zone thickness exceeds four, and layers are deformed such that their lengths are less than four times their width (i.e., not thin flamelets). The percentage of partially distributed layers ranged from 0 to $3 \%$, indicating that distributed regions were not significant in any of the cases studied.

An important quantity for turbulent flames is the likelihood of local extinction; the regime diagram predicted in Figure 1 suggests that at sufficient turbulence the reaction zones can no longer resist the strain of the flow and will either fully thicken into distributed reactions or break apart (broken reactions). 


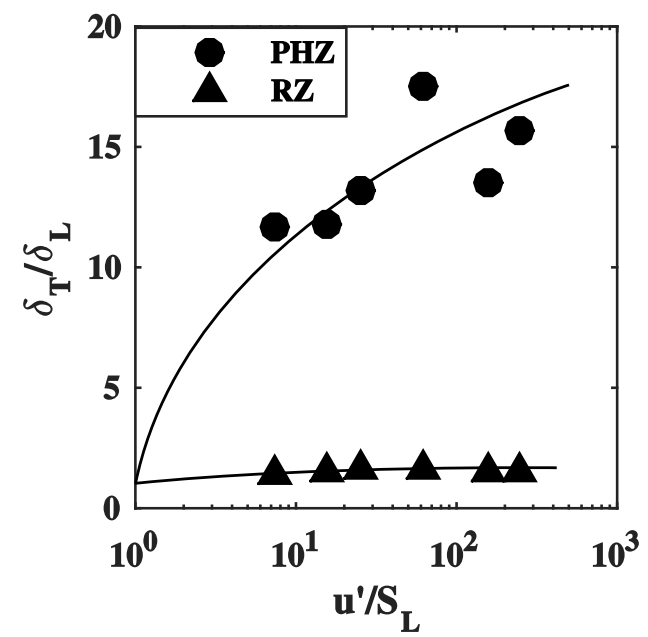

Figure 5: Average preheat zone and reaction zone thicknesses normalized by the laminar zone thickness as computed in CHEMKIN as a function of $u^{\prime} / S_{L}$.

This was quantified in the present data using the burning fraction. The burning fraction is defined as the ratio of reaction zone length to premixed reactants surface length in a given image. Formaldehyde signal is used as the marker for the premixed reactants surface, and care is taken to only select the flame-side edge. This is done by segmenting the edges of the binarized $\mathrm{CH}_{2} \mathrm{O}$ image into seperate objects, and discaring the segments that are both on the jet-exit side of the field of view and are more than one laminar flame thickness away from an $\mathrm{OH}$ boundary. The quantity is ensemble-averaged over all frames to derive a representative amount of local extinction for each test case. The authors have outlined the processing steps involved in calculating the burning fraction in Ref. [26].

The burning fraction was evaluated and found to be nearly constant at all conditions, with no cases falling below 0.98 (indicating less than $2 \%$ extinction). Therefore, none of the cases studied can be said to have broken reaction zones. Large-scale extinction in the Hi-Pilot was only possible when using a less robust co-flow. This allowed cold, ambient, room air to be entrained into the turbulent jet, quenching chemical reactions. All test cases in the present study used a sufficiently large co-flow to sheathe the flame with hot products, and the burning fraction was not computed for a condition with a weaker co-flow producing global extinction. However, large FOV formaldehyde and OH-PLIF images were recorded with a reduced co-flow and showed an open flame tip, indicated by the formaldehyde signal persisting downstream far beyond the trailing edge of OH-PLIF signal. Kiefer et al. [36] used simultaneous CH and OH-PLIF and observed identical results. This highlights the importance of co-flow geometry and temperature in maintaining combustion in extreme turbulence. 


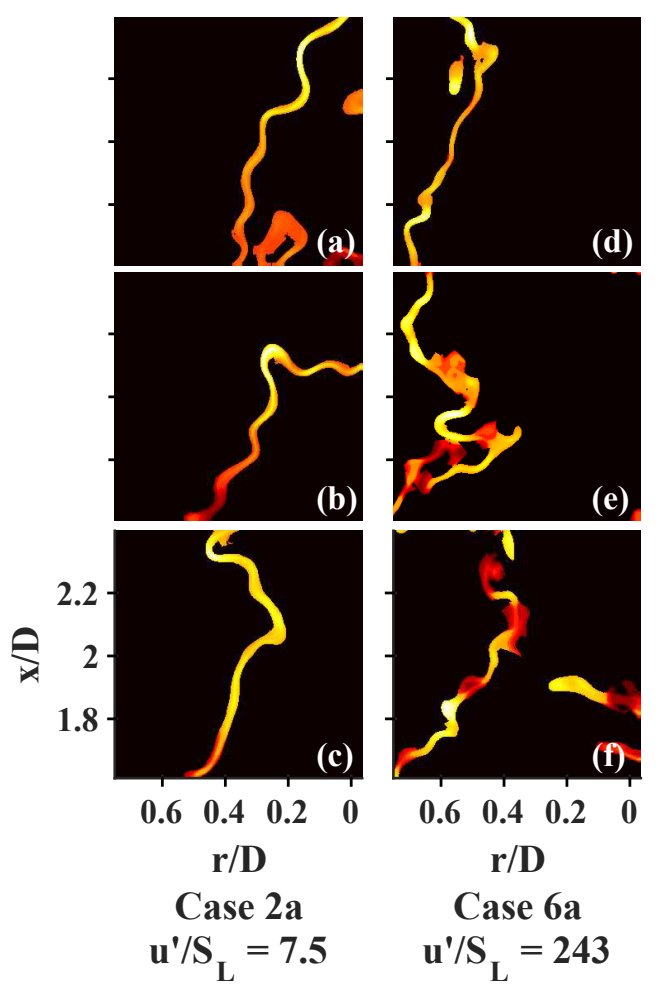

Figure 6: Reactions zones for the Case $2 \mathrm{a}(\phi=1.05$, panels a-c) and Case $6 \mathrm{a}(\phi=0.65$, panels d-f), at three random times.

The present work indicates some limited broken reaction zones (see Figure $6(f)$ ), however these instances occur on average in less than $2 \%$ of the flame surface. Peters has predicted [16] that as the turbulence intensity is increased beyond a critical value (see BR boundary in Figure 1), broken reactions will occur. However, the present work suggests this theory requires examination, as two of the test cases fell well above the predicted BR boundary, yet no significant change in flame structure was observed. There are several possible explanations for this discrepancy. The regime boundaries as drawn by Peters contain several assumptions regarding both the turbulence and flame structure, which simplify the analysis but may not be valid. Additionally, the criterion for broken or distributed reactions $\left(\delta_{R Z}=\eta_{K}\right)$ does not account for the attenuation of turbulence that will occur through a broadened preheat zone. Preheat zones greater than $20 \mathrm{~mm}$ in thickness (as observed in the Hi-Pilot) will present a large region of elevated viscosity across which turbulence will decay. The ratio of turbulence conditions ( $u^{\prime}$ and $L_{x}$ ) measured upstream of the preheat layer and measured at the reaction front is unknown; however, evaluation of the Kolmogorov scale must account for attenuation of the turbulence by the preheat 
layer.

Based on the present results, it appears that this phenomenon makes achieving fully broken or distributed reaction layers much more difficult than previously believed; as the turbulence is increased, the preheat zone thickens progressively, which increasingly reduces the effective turbulence acting on the reaction front. This implies that the turbulence required at the jet exit must be significantly larger than previously believed. If condition ' 0 ' describes the jet exit and '*' describes conditions at the reaction front, we require $\eta_{k}^{0} \lll \delta_{R Z}$, so that $\eta_{k}^{*}=\delta_{R Z}$. The exact relation between $\eta_{k}^{0}$ and $\eta_{k}^{*}$ remains a research question. It is postulated that fully broken reaction zones would require a significant increase in turbulence intensity in order to overcome this effect.

One objection that could be raised is the turbulence at the jet exit is decaying downstream, and therefore the turbulence conditions at the flame front may in fact be in the predicted BP-TR regime, and thus agree with the measurements. Therefore, it is an open question what value of turbulence should be used when considering a regime diagram. The measurement most consistent with Peters' theory would be to measure the local turbulence level at the reaction front, averaged over the entire flame surface. The regime diagram was conceived assuming turbulent eddies interacting normally with the flame, which would require the true turbulence statistics at the flame front. This would account for turbulent decay, as well as attenuation through the preheat zone which has been discussed previously. However, this has little use for practical applications; instead, boundary conditions such as bulk flow velocity, inlet centerline velocity and turbulence intensity, and turbulence length scales should be used. Regime boundaries should be modified to account for any real-world effects (such as decay and attenuation), but use of a local (instead of global) variable defeats the purpose of a regime diagram, which is to predict combustion characteristics given a reduced set of boundary variables and initial conditions.

\section{Conclusions}

a) A new burner (Hi-Pilot) provides turbulence levels and integral scales in a new range that is called extreme turbulence. Previous studies with turbulent Bunsen flames considered $u^{\prime} / S_{L}$ up to 24 ; Hi-Pilot extends the range of $u^{\prime} / S_{L}$ by a factor of ten - up to 243 . Integral scale was systematically varied by a factor of six (from 6 to $41 \mathrm{~mm}$ ) and turbulent Reynolds number was as large as 99,000.

b) Overlap PLIF diagnostics (involving hydroxyl and formaldehyde signals) were assessed using a laminar calibration flame and were found to image the preheat and reaction zones with good signal-to-noise ratios.

c) The average preheat zone thickness increased with $u^{\prime} / S_{L}$ until it reached 16 times the laminar value in this new range of extreme turbulence.

d) In contrast, reaction zone thickness did not increase appreciably with turbulence level and was typically less than twice the laminar thickness. It is believed that after eddies broaden the preheat zone, they can no longer reach the reaction zone due to viscous decay. 
e) For four cases the measured regime was found to agree with the predicted regime. These cases had a Broadened Preheat - Thin Reaction layer (BPTR) structure. However, two other cases lie in the predicted regime of Broken Reactions (BR) yet the measurements indicate that they are not broken and remain in the BP-TR regime. It is concluded that the predicted BR boundary of Peters is not quantitatively correct, and may need modification as more experimental evidence is reported.

f) The preheat layer is thin near the flame base but thickens increasingly at downstream (x) locations, in proportion to the characteristic residence time $(\mathrm{x} / \mathrm{U})$. Thus the residence time appears to be an important parameter governing flame structure, although it is ignored in most regime diagram formulations. However, the reaction zone thickness does not appear to increase with $\mathrm{x} / \mathrm{U}$. Therefore the turbulence that is incident on the preheat layer may differ from turbulence incident on the reaction layers.

g) Broken reactions, fully distributed, and partially-distributed reaction zones were not observed for any of the six cases, even for extreme turbulence levels in the reactants of $u^{\prime} / S_{L}=243$. One possible explanation is that the relevant values of turbulence, Karlovitz, and Reynolds numbers may be those that occur at the reaction layer. These values are expected to be smaller than those in the reactants due to viscous attenuation within the very thick preheat layers in this new range of turbulence.

h) It is not yet possible to map out the measured boundaries of the Borghi diagram until more data are collected, although the present results indicate the predicted boundary for broken reaction zones requires examination. A cautious approach is to assume that the present results apply only to piloted Bunsen flames until additional measurements are reported for other flame geometries.

\section{Acknowledgments}

The authors gratefully acknowledge Drs. Carter and Hammack for their insightful comments and advice in the preparation of this manuscript. Support

for this research was provided by AFOSR Grant FA9550-12-1- 0101; additional support was provided by National Science Foundation grant CBET 0852910.

\section{References}

[1] E. Knudsen, and H. Pitsch, Combust. Flame 159 (2012) 242-264.

[2] R. Sankaran, E.R. Hawkes, C.S. Yoo, J.H. Chen, Combust. Flame 162, 9 (2015) 3294-3306

[3] A. J. Aspden, M. S. Day, J. B. Bell, J. Fluid Mech. 680 (2011) 287-320.

[4] B. Savard, B. Bobbitt, G. Blanquart, Proc. Combust. Inst. 35, 2 (2015),1377-1384. 
[5] B. Sundaram, A.Y. Klimenko, M.J. Cleary, U. Maas, Proc. Combust. Inst. 35 (2015) 1517-1525.

[6] K. Huh, J. Kwon, D. Lee, Physics of Fluids 25, 7 (2013) 075108.

[7] D. Lee, K. Y. Huh, Combust. Flame 159, 4 (2012) 1576-1591

[8] F. Yuen, O. Gülder, Comb. Sci. Tech. 182 (2010) 544-558

[9] P. Tamadonfar, Ö. L. Gülder, Comb. Flame 161 (2014) 3154-3165

[10] D. Bradley, M. Lawes, K.X. Liu, M.S. Mansour, Proc. Combust. Inst. 34, 1, (2013) 1519-1526.

[11] D. Bradley, M. Lawes, M.S. Mansour, Flow Turb. Combust. 87, 2-3 (2011) 191-204.

[12] F. Dinkelacker, A. Soika, D. Most, D. Hoffman, A. Leipertz, W. Polifke, K. Dobbeling, Proc. Combust Inst. 27 (1998) 857-865

[13] F. Dinkelacker, S. Pfadler, A. Leipertz, Z. Phys. Chem. 223 (2009) 481-502

[14] J. Kariuki, J.R. Dawson, E Mastorakos, Combust. Flame 159, 8 (2012) 2589-2607.

[15] R. Borghi, Prog. Energy Combust. Sci. 14 (4) (1988) 245-292.

[16] N. Peters, Turbulent Combustion, Cambridge U. Press, Cambridge UK, 2000.

[17] N. Peters, AIAA (1991) 155-182 (A 92-16977 04-25)

[18] F. A. Williams, Combust. Flame 26 (1976) 269-276.

[19] M.J. Dunn, A. R. Masri, R. W. Bilger, R S. Barlow, Flow Turbulence Combust. 85 (2010) 621-648.

[20] B. Zhou, C. Brackmann, Q. Li, Z. Wang, P. Petersson, Z. Li, M. Alden, X. Bai, Combust. Flame, 162 (2015) 2937-2953.

[21] M. S. Sweeney, S. Hochgreb, M. J. Dunn, R. S. Barlow, Combust. Flame 159 (2012) 2896-2911.

[22] M.S. Mansour, Y.-C. Chen, N. Peters, Proc. Combust. Inst. 24 (1992) 461468.

[23] I.G. Shepherd, R. K. Cheng, Combust. Flame 127 (2001) 2066-2075.

[24] A. Buschmann, F. Dinkelacker, T. Schafer, M. Schafer, J. Wolfrum, J.. Proc. Combust. Inst. 26 (1996) 437-445.

[25] W. Meier, X.R. Duan, P. Weigand, Proc. Combust. Inst. 30 (2005) 835-842 
[26] J. E. Temme, T. M. Wabel, A. W. Skiba, J. F. Driscoll, 53rd AIAA Aerospace Sciences Meeting, AIAA Paper 2015-0168 (2015).

[27] Li, B. Li, Z. Sun, X. Bai, M. Alden, Combust. Flame 157 (2010) 1087-1096.

[28] B.O. Ayoola, R. Balachandran, J.H. Frank, E. Mastorakos, C.F. Kaminski, Combust. Flame 144 (2006) 1-16.

[29] S. Böckle, J. Kazenwadel, T. Kunzelmann, D-I. Shin, C. Schulz, J. Wolfrum. Proc. Combust. Inst. 28 (2000) 279-286

[30] M. Röder, T. Dreier, C. Schulz, Proc. Combust. Inst. 34 (2013) 3549-3556.

[31] B. Peterson, E. Baum, B. Bohm, A. Dreizler, Proc. Combust. Inst. 35 (2015) 3829-3837

[32] H. N. Najm, P. H. Paul, C. J. Mueller, P. S. Wyckoff, Combust. Flame 113 (1998) 312-332Z.

[33] P. Hrycak, D. T. Lee, J. W. Gauntner, J. N. B. Livingood, NASA Technical Note D-5690, March 1970

[34] A. Marshall, P. Venkateswaran, D. Noble, J. Seitzman, T. Lieuwen, Expt. Fluids 51 (2011) 611-620.

[35] T. M. Wabel, A. W. Skiba, J. F. Driscoll, Proc. Combust. Inst. 36 (2016) In print

[36] J. Kiefer, Z. S. Li, J. Zetterburg, X. S. Bai, M. Alden, Combust. Flame 154 (2008) 802-818 